\title{
A New Intrinsic Magnetic Property Due to the Mesoscopic Ordering of Cobalt Nanocrystals in Supra-Crystals
}

\author{
D. Parker, C. Salzemann and I. Lisiecki*
}

\author{
Laboratoire des Matériaux Mésoscopiques et Nanométriques, UMR CNRS 7070, Université P. et M. Curie Bât F, 4 \\ Place Jussieu, 75005 Paris, France
}

\begin{abstract}
Recently, it has been shown that nanoparticles with a narrow size distribution can self-organize into highly ordered fcc supra-crystals. This new generation of materials have been found to exhibit unique collective properties, which arise from the periodic ordering of the nanoparticles. Using the expertise we have to control the ordering of cobalt nanoparticles in 3D assemblies, we have been able to carry out a carefully controlled comparative investigation of the magnetic properties of a supracrystal and a disordered assembly. In this letter, we report for the first time a new intrinsic effect of supra-crystal order on the magnetic behavior which we attribute to a decrease in the distribution of energy barriers, arising from nanoparticle ordering. We also show that these assemblies can be annealed at $350^{\circ} \mathrm{C}$ to give a structural transition of the Co nanoparticles from an fcc to hcp phase. This annealing does not induce either coalescence or oxidation of the nanocrystals.
\end{abstract}

\section{INTRODUCTION}

In recent years, several studies have been carried out on the behavior of magnetic nanoparticles arranged in 2 and $3 \mathrm{D}$ assemblies [1-2]. As yet, no conclusive evidence of an effect of nanoparticle order on the collective magnetic properties has been observed. As atoms arranged differently in solids exhibit different physical properties, e.g. carbon arranged in an amorphous solid (graphite) or in an fcc structure (diamond), we can expect physical differences when nanocrystals are arranged in a disordered fashion or in a highly ordered supra-structure. For example, supracrystals made of silver nanocrystals have recently been shown to exhibit vibrational coherence in Raman scattering [3]. Enhanced mechanical stability of columns made of cobalt nanocrystals has been shown to arise from the fcc supra-structure [4]. In addition, it was shown that there is a change in the photoluminescence properties between ordered and disordered 3D assemblies of CdSe nanocrystals [5] Using the expertise we have to control the ordering of cobalt nanocrystals in 3D assemblies [6], we report for the first time, an intrinsic effect of supra-crystal order on the magnetic behavior, which we attribute to a decrease in the distribution of energy barriers and an increased sample anisotropy arising from nanocrystal ordering.

It is well known that magnetic particles of sufficiently small size $(<50 \mathrm{~nm})$ are monodomain and hence behave as a single giant spin. This enables interesting comparisons to be drawn between mesoscopic and atomic magnetic systems and magnetic behavior ranging from superparamagnetism to spinglass-like can be observed [7-8]. Moreover, increasing the magnetocrystalline anisotropy of the nanocrystals in an assembly (for example by annealing [9-10]) can optimize the interactions in the system leading to magnetic transitions close to room temperature, which are desirable for applications.

*Address correspondence to this author at the UPMC, Laboratoire LM2N, Bat F74 porte 633, 4 place jussieu, 75005 Paris, France; Tel: 01442725 17; Fax: 01442725 15; E-mails: lisi@sri.jussieu.fr, lisiecki@ccr.jussieu.fr
We have developed synthetic and deposition procedures which allow us to fabricate, with the same population of nanocrystals, either disordered or long-range fcc ordered 3D assemblies [6]. This enables us to ensure that any differences observed in the magnetic properties arise uniquely from the change in ordering of the $3 \mathrm{D}$ assemblies. As well as controlling the mesoscopic order of the 3D assemblies we can also control, by annealing, the atomic order of the Co nanoparticles.

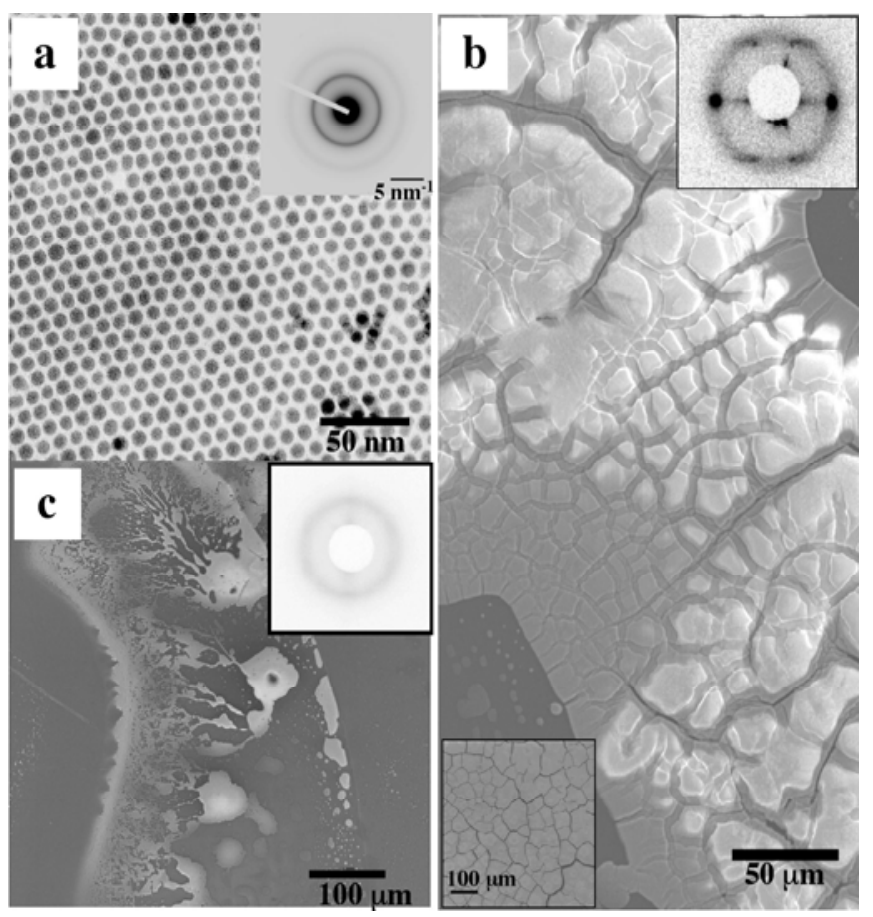

Fig. (1). Native $3 D$ assemblies. (a) TEM images of $7.5 \mathrm{~nm}$ cobalt nanocrystals ordered in a compact hexagonal network. Insert: Corresponding electron diffraction pattern. (b) SEM images of a supracrystal. Insert top: Corresponding GISAXS pattern and insert bottom: SEM image illustrated the thicker film. (c) SEM images of a disordered 3D assembly. Insert: Corresponding GISAXS pattern. 
The cobalt nanocrystals used for this study are characterized by a mean diameter and size polydispersity of $7.5 \mathrm{~nm}$ and $9.4 \%$ respectively and are stabilized by a coating of dodecanoic acid chains (Fig. 1a) [11]. The electronic diffraction image (inset, Fig. 1a) indicates fcc polycrystals. The ordered and disordered 3D assemblies are prepared on HOPG substrates [6]. As shown in the scanning electron microscopy (SEM) image (Fig. 1b), the ordered 3D assembly has either a step-like, largely un-cracked surface (Fig. 1b) or a cracked surface (inset, bottom of Fig. 1b). The corresponding grazing incidence small-angle X-ray scattering (GISAXS) pattern shows reflections characteristic of a high order of the nanocrystals in a fcc mesoscopic structure growing in the [111] direction (inset, top of Fig. 1b) [12]. From the (111) reflection we deduce a periodicity of the lying planes equal to $8.6 \mathrm{~nm}$. This value gives us a centre-to-centre interparticle distance $\left(D_{c-c}\right)$ equal to $10.5 \mathrm{~nm}$, which in turn gives an interparticle gap $\left(D_{i-p}\right)$ of $3.0 \mathrm{~nm}$ (Table 1). The interdigitation of the alkyl chains (estimated to be around 6 C-C distances) is the basis for the high structural stability of the supra-crystals [13].

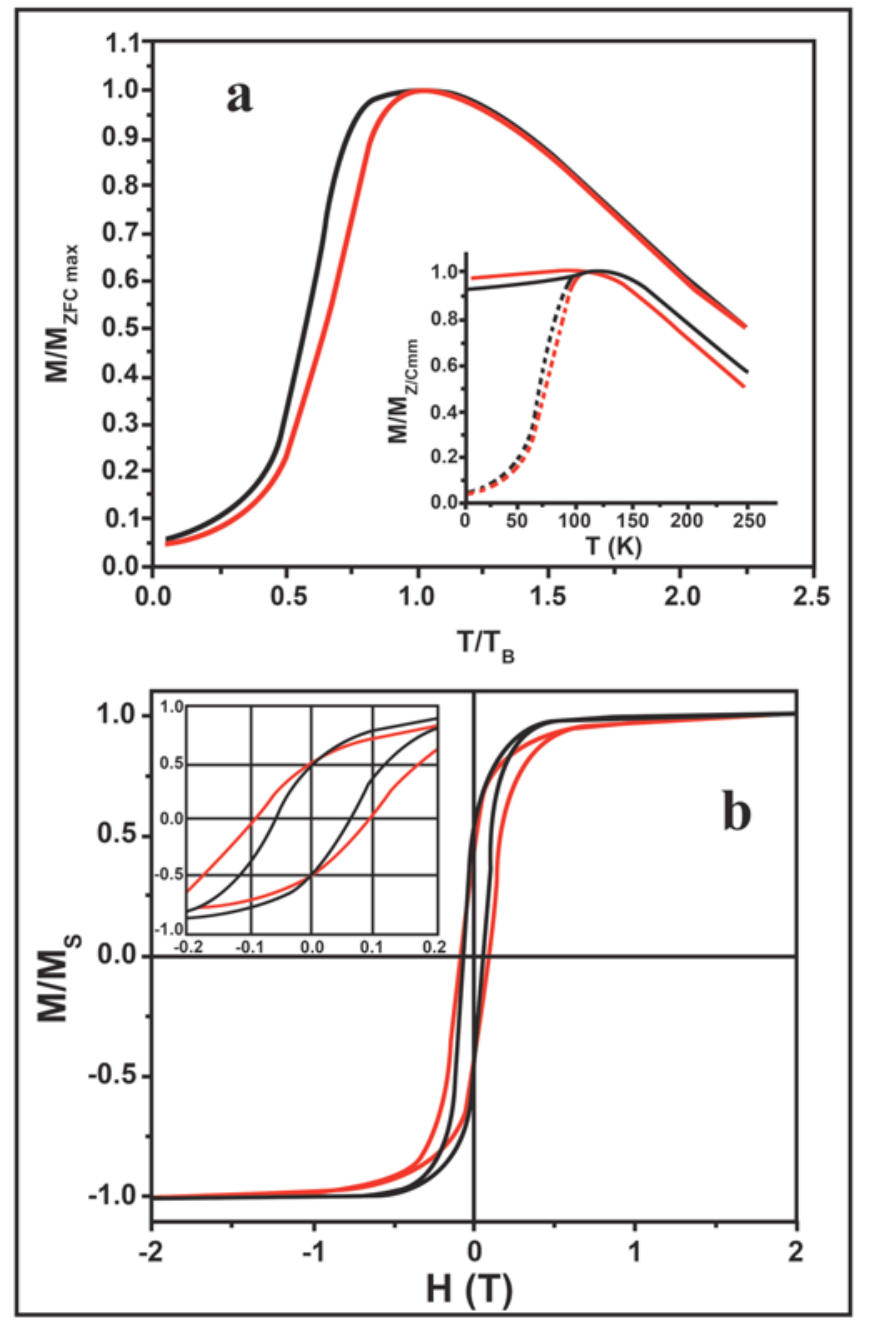

Fig. (2). (a) ZFC $M$ versus $T / T_{B}$ curves of disordered (black line) and ordered (red line) 3D assemblies. Insert: The raw ZFC (dashed line) and FC (full line) $M$ versus $T$ curves. (b) $M$ versus $H$ curves of disordered (black line) and ordered (red line) 3D assemblies. Insert: Enlargement of the low field region.
Fig. (1c) shows the SEM image of the disordered 3D assembly; the film consists of smooth aggregates, highly polydisperse in size and shape. The GISAXS pattern (inset, Fig. 1c) shows a diffuse ring indicating a disordered sample. The estimate $D_{i-p}$ is of $4.0 \mathrm{~nm}$.

Table 1. Structural and Magnetic Parameters Extracted from the GISAXS Patterns

\begin{tabular}{|c|c|c|c|c|}
\hline \multirow{2}{*}{$\begin{array}{c}\text { Annealing } \\
\text { Temperature }\end{array}$} & \multicolumn{2}{|c|}{ Native } & \multicolumn{2}{c|}{$350^{\circ} \mathrm{C}$} \\
\cline { 2 - 5 } & Ordered & Disordered & Ordered & Disordered \\
\hline \hline $\begin{array}{c}(111) \text { stacking } \\
\text { periodicity (nm) }\end{array}$ & $8.6 \pm 0.1$ & $9.4 \pm 0.1$ & $7.9 \pm 0.1$ & $9.1 \pm 0.1$ \\
\hline$\delta \mathrm{q}_{1 / 2}\left(\mathrm{~nm}^{-1}\right)$ & 0.050 & 0.260 & 0.040 & 0.240 \\
\hline$D_{c-c}(\mathrm{~nm})$ & $10.5 \pm 0.1$ & $11.5 \pm 0.1$ & $9.7 \pm 0.1$ & $11.2 \pm 0.1$ \\
\hline$D_{i-p}(\mathrm{~nm})$ & $3.0 \pm 0.5$ & $4.0 \pm 0.5$ & $2.2 \pm 0.5$ & $3.7 \pm 0.5$ \\
\hline$T_{B}(\mathrm{~K})$ & $112 \pm 3$ & $120 \pm 8$ & $280 \pm 3$ & $265 \pm 3$ \\
\hline$M_{r} / M_{s}$ & $0.53 \pm 0.2$ & $0.54 \pm 0.2$ & $0.51 \pm 0.2$ & $0.52 \pm 0.2$ \\
\hline$M_{\text {snat }} / M_{\text {sann }}$ & - & - & $0.63 \pm 0.2$ & $0.78 \pm 0.2$ \\
\hline$H_{c}(\mathrm{Oe})$ & $900 \pm 50$ & $600 \pm 50$ & $900 \pm 50$ & $800 \pm 50$ \\
\hline
\end{tabular}

The ZFC magnetization curves and the hysteresis. $\delta \mathrm{q}_{1 / 2}$ : the half width at half maximum; $D_{c-c}$ : center to center NC distance; $D_{i-p}$. : border to border distance of NCs considering a NC size of $7.5 \mathrm{~nm} ; T_{B}$ : blocking temperature; $M_{s}$ : saturation magnetization; $M_{\text {snat }} / M_{\text {sann }}$ : ratio of native $M_{s}$ to annealed $M_{s} ; H_{c}$ : coercivity.

In order to characterize the magnetic behavior of the disordered and supra-crystal samples, we first investigated the low-field magnetic properties. Fig. (2a) shows the zero field cooled (ZFC) magnetization versus temperature curves measured with an applied field of $20 \mathrm{Oe}$, of both the ordered and disordered $3 \mathrm{D}$ assemblies, normalized to the blocking temperature $\left(T_{B}\right)$. The raw field cooled $(\mathrm{FC})$ and $\mathrm{ZFC}$ curves are shown in the insert. For both samples, $T_{B}$ is of around $100 \mathrm{~K}$, indicative of strong interparticle interactions as expected for a $3 \mathrm{D}$ assembly where the interparticle distances are small. There is no significant difference in $T_{B}$ between the ordered and disordered assemblies, however the width of the normalized ZFC peak is greater for the disordered sample than for the supra-crystal samples. This feature was found to be highly reproducible in several pairs of ordered and disordered samples. The width of the ZFC peak is related to the distribution of energy barriers in the system, the main constituents of which are the activation energies, $E_{a}=$ $k_{a} V$ (where $k_{a}$ is the anisotropy constant and $V$ the particle volume) and the dipole-dipole interaction energies, $E_{d d}[14]$. As the same nanoparticles are used to build both the ordered and disordered assemblies, the distribution of $k_{a}$ and $V$ will be the same in both cases and hence will not lead to a change in ZFC peak width. However, as pointed out in [15], $E_{d d}$ is highly directionally dependent and therefore the geometrical arrangement of the nanoparticles in the 3D assembly will influence the distribution of $E_{d d}$. Consequently, in the supracrystal sample, where the nanoparticles are arranged in a regular, ordered fashion, the distribution of $E_{d d}$ and therefore the width of the ZFC peak will be narrower than in the disordered 3D assembly where the nanoparticles are either totally disordered or ordered on short range only.

The magnetic behavior of these assemblies was further investigated by studying the high field magnetization curves. 
As shown in Fig. (2b), both samples show hysteresis at $5 \mathrm{~K}$ and reach magnetic saturation at around $1 \mathrm{~T}$. Some differences can be observed between the ordered and disordered samples, namely an increased coercive field $\left(H_{c}\right)$ and a slower approach to magnetic saturation for the supra-crystal w.r.t the disordered assembly. This faster approach to saturation seen for the disordered sample is coherent with a $\Delta M \approx$ $H^{-1 / 2}$ behavior as observed in amorphous ferromagnets [16]. Experimental studies on these materials and on analogous nanoparticle films have shown that a higher anisotropy leads to a slower approach to saturation as we observe for the supra-crystal sample [17-18]. We therefore propose that the supra-crystal has a higher sample anisotropy compared to the disordered sample (as is also confirmed by the higher $H_{c}$ ) due to the increased mesoscopic order. This behavior presents an analogy with bulk crystalline materials where higher atomic crystallographic order leads to an increase in anisotropy.

We now show that by annealing the Co nanocrystals at $350^{\circ} \mathrm{C}$ in situ in the $3 \mathrm{D}$ assemblies, we can induce the improvement of both the crystallographic structure of the nanocrystals and that of the mesoscopic structure of the supra-crystal. As illustrated by the electronic diffraction pattern (inset, Fig. 3a), annealing drastically improves the nanocrystal crystallinity from a poorly crystallized fcc structure to a monocrystalline hcp structure [9]. No oxidation is detected. As can be seen in the insert of Fig. (3b), the GISAXS pattern of the ordered sample shows that the fcc mesostructure is maintained without any observable coalescence. In addition, the (111) stacking periodicity decreases by about $0.8 \mathrm{~nm}$ (Table 1); we attribute this to a decrease in $D_{i-p}$. In parallel, a decrease in the HWHM (Half Width at Half Maximum) of the (111) reflection, $\delta q_{1 / 2}$, is found indicating an increase in the coherence length compared to the native sample. These features arise due to an increase in the mobility of the alkyl chains during annealing [19] allowing the nanocrystals to rearrange slightly, improving the mesoscopic order. As a consequence of the lattice shrinking, we observe the appearance of cracks in the thinner region of the film (see SEM image, Fig. 3b)

As can be seen from the GISAXS pattern of the annealed disordered sample (inset Fig. 3c), no new reflections appear after annealing, indicating the absence of long-range order. The deduced $D_{i-p}$ indicates a slight contraction of the lattice $(\approx 0.3 \mathrm{~nm})$ that could suggest a local improvement of the nanocrystal order, not detectable through new reflections. While we observe the formation of some cracks in the film, there is no significant change in morphology (see Fig. 3c).

Fig. (4a) shows the ZFC magnetization versus temperature curves normalized to $T_{B}$ for the ordered and disordered samples after annealing at $350^{\circ} \mathrm{C}$. The insert shows the raw FC and ZFC curves for the same samples. We see that for both samples the $T_{B}$ has increased significantly (see Table 1) and we attribute this to an increase in the anisotropy of the Co nanocrystals, arising from the change in crystallinity from fec to hep nanocrystals. This is consistent with the behavior observed for $2 \mathrm{D}$ and diluted assemblies of Co nanocrystals [9] where a 3-fold increase in $K_{a}$ was found after the fcc-hcp transition. After annealing, the width of the supracrystal normalized ZFC peak remains unchanged w.r.t. the native sample whereas for the disordered sample a significant narrowing is observed.

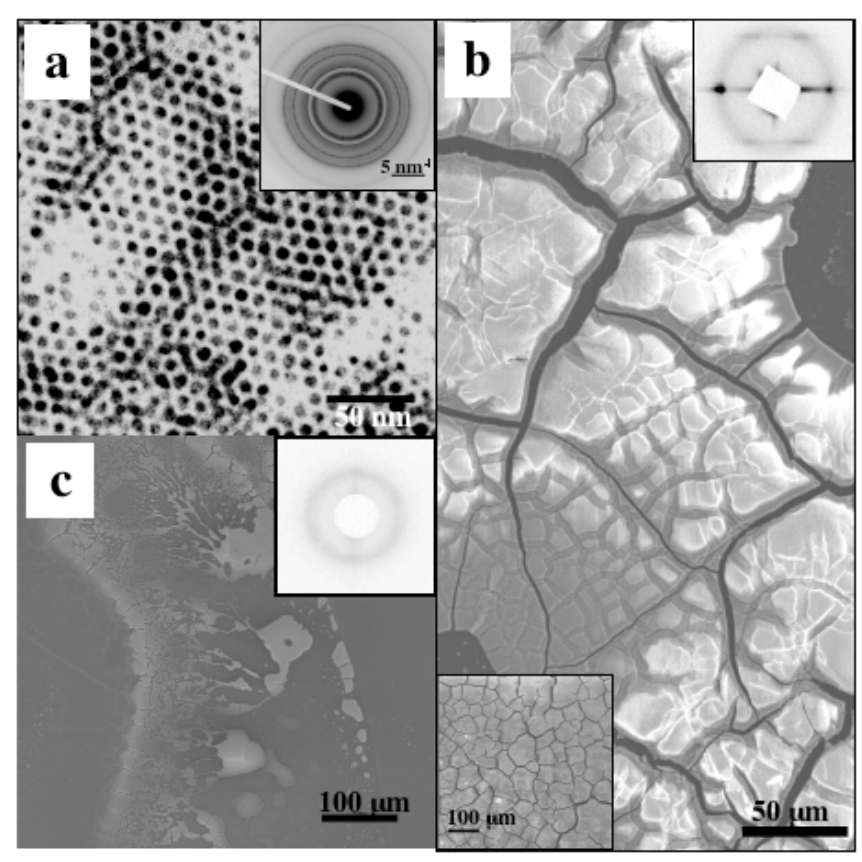

Fig. (3). $3 D$ assemblies annealed at $350^{\circ} \mathrm{C}$. (a) TEM images of 7.5 $\mathrm{nm}$ cobalt nanocrystals ordered in a compact hexagonal network. Insert: Corresponding electron diffraction pattern. (b) SEM images of a supra-crystal. Insert top: Corresponding GISAXS pattern and insert bottom: SEM image illustrated the thicker film. (c) SEM images of a disordered 3D assembly. Insert: Corresponding GISAXS pattern.

The normalized ZFC curves of the annealed disordered and ordered samples are now superimposed. For both samples there is a significant increase in saturation magnetization $\left(M_{S}\right)$ after annealing due to the formation of pure hcp nanocrystals [9]. (NB, due to the very low mass of these 3D assemblies with respect to their substrates, accurate determination of the Co mass was not possible and therefore only relative and not absolute values of $M_{s}$ can be determined.) In the native sample the nanocrystals are polycrystalline, leading to a net moment that is the sum of the moments of the randomly orientated magnetic domains. After annealing, the nanoparticle is monocrystal with a single magnetic domain and therefore the net magnetic moment is larger than for the native nanocrystals. We therefore attribute the increase in $M_{s}$ largely to the change from polycrystalline nanoparticles to monocrystals rather than to the change from fcc to hcp atomic structure.

The absence of a peak at around $8 \mathrm{~K}$ in the $\mathrm{ZFC}$ curve further confirms the absence of oxidation of the metallic material.

The magnetization versus field curves, measured at $5 \mathrm{~K}$, of the annealed ordered and disordered samples (Fig. 4b) show that the $H_{c}$ of the ordered sample remains unchanged after annealing whereas that of the disordered sample increases (Table 1). Therefore, after annealing, the differences observed between the native ordered and disordered samples (a narrower ZFC peak and increased $H_{c}$ for the ordered sample) are no longer observed. We propose that after annealing the relative importance of the nanocrystal anisotropy and the 
dipolar interactions has changed and, as studied theoretically in reference [15], this can significantly alter the magnetic behavior thus obscuring the effects of supra-crystal order.

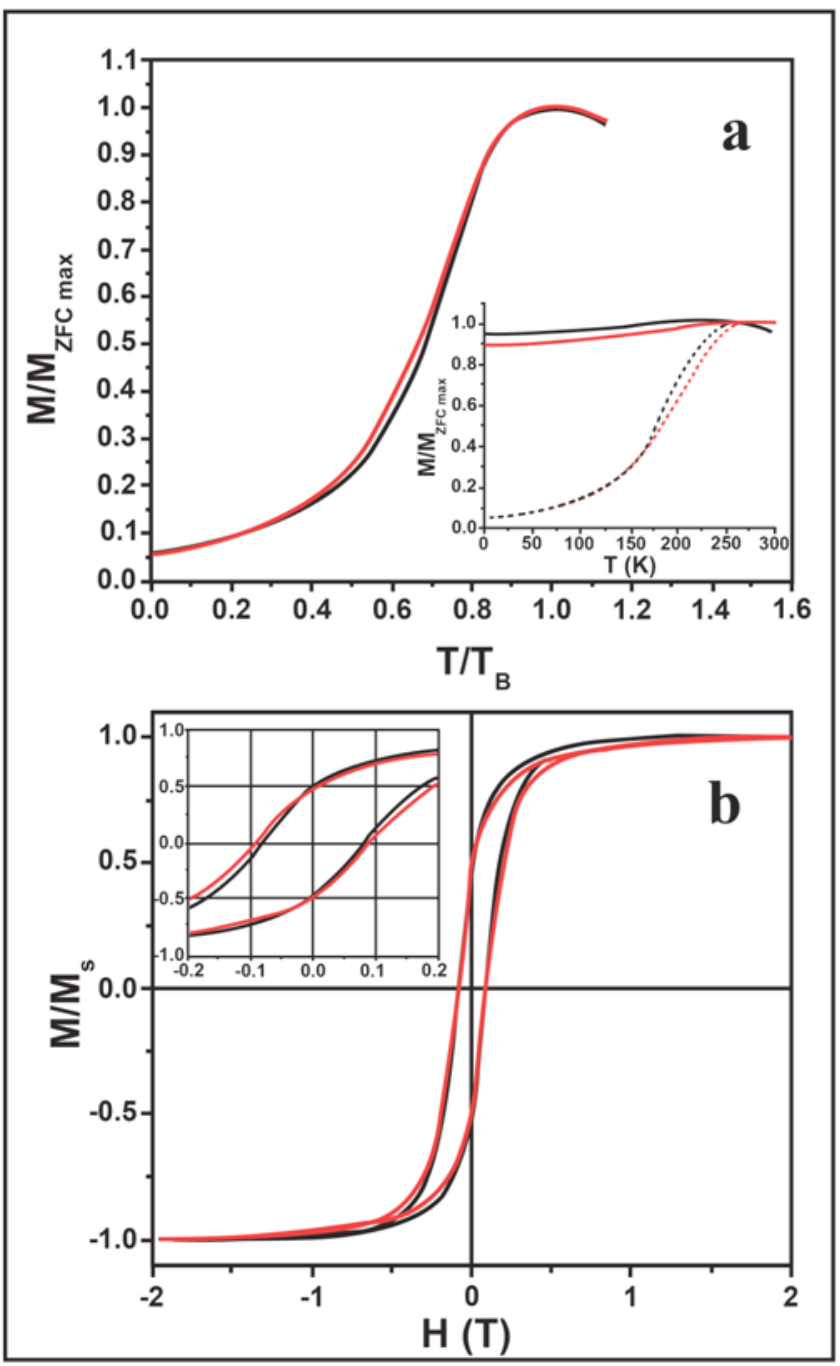

Fig. (4). (a) ZFC $M$ versus $T / T_{B}$ curves of disordered (black line) and ordered (red line) 3D assemblies. Insert: The raw ZFC (dashed line) and FC (full line) $M$ versus T curves. (b) $M$ versus $H$ curves of disordered (black line) and ordered (red line) 3D assemblies. Insert: Enlargement of the low field region.

In summary, we have found that by annealing supracrystals with an fcc mesoscopic structure at $350^{\circ} \mathrm{C}$, we can induce an atomic structural transition in $7.5 \mathrm{~nm}$ Co nanocrystal building blocks from fcc polycrystals to hep monocrystals. Importantly, such thermal treatment does not destroy the fcc supra-structure; neither oxidation nor coalescence of the nano-material occurs. This point is not trivial as many studies concerning the annealing of suprastructures have reported instability due to these two factors [20-22]. In our case, we actually observe an increase in the coherence length of the mesoscopic structure of the 3D superlattice. In addition, we have found for the first time that supra-crystal order has an effect on the magnetic properties of a 3D Co nanocrystal assembly, arising from the narrow distribution of $E_{d d}$. After annealing, we see a drastic increase in both $T_{B}$ and $M_{s}$ arising from the higher anisotropy and crystallinity associated with the pure hop structure. The differences in the magnetic behavior of the ordered and disordered assemblies are no longer observed for the nanocrystal hcp state. This could be explained by an improvement in local mesoscopic order of the disordered sample after annealing, however, we have no concrete evidence to support this. We believe that a more likely explanation arises from the fact that, after annealing, the delicate interplay of dipolar interactions and the single particle anisotropy has been modified and the effects of nanocrystal order are no longer observed.

\section{METHODS}

\section{Synthesis}

Cobalt nanocrystals stabilized by dodecanoic acid chains are synthesized by chemical reduction in reverse micelles (water in oil droplets) as described in a previous paper [11]. The size polydispersity is optimized by controlling the reducing agent (sodium borohydride) concentration. The nanocrystals are dispersed in hexane. Monolayers are obtained by depositing few drops of a cobalt nanocrystal solution on an HOPG coated grid for TEM examination.

The 3D assemblies are obtained by immersing the HOPG substrate of low mass (less than $10 \mathrm{mg}$ ) in $200 \mu 1$ of a nanocrystal solution (5.5 x 10-7 M particles). The ordering of the sample occurs under nitrogen and is controlled by the substrate temperature which is fixed at $5^{\circ} \mathrm{C}$ and $25^{\circ} \mathrm{C}$ for the disordered and ordered samples respectively [6].

The sample annealing at $350^{\circ} \mathrm{C}$ is carried out in a furnace under a flow of nitrogen for $20 \mathrm{mn}$.

\section{Analysis}

Transmission electron microscopy was performed using a JEOL 1011 microscope.

Scanning electron microscopy was performed with a JMS-5510LV microscope.

Grazing Incidence Small-angle X-ray scattering (GISAXS) measurements were carried out using a rotating anode generator operated with a small-size focus (copper anode; focus size $0.2 \mathrm{~mm} \times 0.2 \mathrm{~mm} ; 50 \mathrm{kV}, 30 \mathrm{~mA}$ ). The optics consisted of two parabolic multilayer graded mirror in KB geometry providing a parallel monochromatic beam. The sample was mounted on a rotation stage and the diffraction patterns were recorded on photo-stimulable imaging plates. Vacuum pipes are inserted between the sample and the imaging plate to reduce air scattering. A single GISAXS measurement probes a section, several micrometers wide, from one edge to the other of the substrate. Magnetic measurements were carried out on a Cryogenics Ltd. S600 SQUID magnetometer. The zero field cooled $M$ versus $T$ measurements were carried out by cooling the sample from 300 to 5 $\mathrm{K}$ in zero field then applying a field of 20 Oe and measuring the magnetization while the sample was heated from 5 to 300 $\mathrm{K}$. The field cooled measurements were performed in the same manner with the difference that the field was applied before cooling. The $M$ versus $H$ measurements were performed at $5 \mathrm{~K}$ after zero field cooling. All magnetic measurements were carried out with the applied field parallel to the substrate. 


\section{ACKNOWLEDGEMENTS}

We warmly thank Dr. Eric Vincent and Dr. Pierre Bonville of DRECAM/SPEC, CEA Saclay for the use of their SQUID magnetometer. Authors also thank the European Union for supporting this work project "Growth and supraorganization of transition and noble metal nanoclusters" contract number, NMP4-CT-2004-001594.

\section{REFERENCES}

[1] Batlle, X., Labarta, A. J. Phys. D: Appl. Phys. 2002, 35, R15.

[2] Lin, X-M., Samia A.C.S. J. Magn. Magn. Mater. 2006, 305, 100.

[3] Courty, A, Merme, A., Albouy, P.A., Duval, E. and Pileni, M.P, Nature Material 2005, 4, 395.

[4] Germain, V, Pileni, M.P. Adv. Mat. 2005, 17, 1424.

[5] Zaitseva, N., Dai, Z. R., Leon, F. R. and Krol, D. J. Am. Chem. Soc. 2005, 127, 10221

[6] Lisiecki, I., Albouy, P.A., Pileni, M.P. J. Phys. Chem. B. 2004, $108,20050$.

[7] Dormann, J.L., Fiorani, D., Tronc, E. Adv. Chem. Phys. 1997, 98, 283.

[8] Jonsson, P., Hansen, M.F., Svedlindh, P., Nordblad, P. J. Magn. Magn. Mater. 2001, 226, 1315.
[9] Petit, C., Wang, Z. L., Pileni, M.P. J. Phys. Chem. B 2005, 109, 15309.

[10] Sun S.H., Murray C.B., Weller D., Folks L., Moser A. Science 2000, $287,1989$.

[11] Lisiecki, I. and Pileni, M.P. Langmuir 2003, 19, 9486

[12] Lisiecki, I., Albouy, P.A., Pileni, M.P., Adv. Mater. 2003, 15, 712.

[13] Mukhopadhyay, R., Mitra, S., Pradeep, T., Tsukushi, I., Ikeda, S. J. Chem. Phys. 2003, 118, 4614.

[14] Dormann, J.L., Spînu, L., Tronc, E., Jolivet, J.P., Lucari, F., D’Orazio, F., Fiorani, D. J. Magn. Magn. Mater. 1998, 183, L255.

[15] Kechrakos, D., Trohidou, K.N. Phys. Rev. B 1998, 58, 12169.

[16] Chudnovsky, E. M. J. Appl. Phys. 1988, 64, 5770

[17] Filippi, J. Amaral, V.S., Barbara, B. Phys. Rev. B, 1991, 44, 2842

[18] Thomas, L., Tuaillon, J., Perez, J.P., Dupuis, V., Perez, A., Barbara, B., J. Magn. Magn. Mater. 1995, 437, 140.

[19] Sandhyarani, N., Resmi, M.R., Unnikrishnan, R., Vidyasagar, K. Ma, S., Antony, M.P., Selvam, P.G., Visalakshi, V., Chandrakumar, N., Pandian, K., Tao, Y.T., Pradeep, T. Chem. Mater. 2000, $12,104$.

[20] Sun, S., Murray, C.B. J.Appl. Phys. 1999, 85, 4325.

[21] Yang, H.T., Shen, C.M., Su, Y.K., Yang, T.Z., Gao, H.J., Wang, Y.G. Appl. Phys. Lett. 2003, 82, 4729.

[22] Nie, X., Jiang; J.C., Meletis, E.I., Tung, L.D., Spinu, L. J. Appl. Phys. 2003, 93, 4750 . 